\title{
Radial velocity measurements of B stars in the Scorpius-Centaurus association ${ }^{\star}$
}

\author{
E. Jilinski ${ }^{1,2}$, S. Daflon ${ }^{1}$, K. Cunha ${ }^{1}$, and R. de la Reza ${ }^{1}$ \\ 1 Observatório Nacional/MCT, Rua Gal. Jose Cristino 77, São Cristovão, Rio de Janeiro, Brazil \\ e-mail: jilinski@on.br \\ 2 Main Astronomical Observatory, Pulkovo, St. Petersburg, Russia
}

Received 7 July 2004 / Accepted 30 October 2005

\section{ABSTRACT}

We derive single-epoch radial velocities for a sample of 56 B-type stars members of the subgroups Upper Scorpius, Upper Centaurus Lupus and Lower Centaurus Crux of the nearby Sco-Cen OB association. The radial velocity measurements were obtained by means of high-resolution echelle spectra via analysis of individual lines. The internal accuracy obtained in the measurements is estimated to be typically $2-3 \mathrm{~km} \mathrm{~s}^{-1}$, but depends on the projected rotational velocity of the target. Radial velocity measurements taken for 2-3 epochs for the targets HD 120307, HD 142990 and HD 139365 are variable and confirm that they are spectroscopic binaries, as previously identified in the literature. Spectral lines from two stellar components are resolved in the observed spectra of target stars HD 133242, HD 133955 and HD 143018, identifying them as spectroscopic binaries.

Key words. stars: early-type - stars: binaries: spectroscopic - stars: kinematics - techniques: radial velocities open clusters and associations: individual: Scorpius-Centaurus association

\section{Introduction}

The Scorpius-Centaurus association is the nearest association of young OB stars to the Sun. Blaauw $(1960,1964)$ divided this association into three stellar subgroups: Upper Scorpius (US), Upper Centaurus Lupus (UCL) and Lower Centaurus Crux (LCC). LCC and UCL have roughly similar ages of about 16-20 Myr, while US is younger with an estimated age of 5 Myr (Mamajek et al. 2002; Sartori et al. 2003). This complex OB association of unbound stars is of great interest because, as recently shown, it is related to the origins of nearby moving groups of low mass post-T Tauri stars with ages around 10 Myr: the $\beta$ Pictoris Moving Group, the TW Hydra association, and the $\eta$ and $\epsilon$ Chamaleonis groups (Mamajek et al. 2000; Ortega et al. 2002, 2004; Jilinski et al. 2005). In addition, the Scorpius-Centaurus association also appears to be the source of a large bubble of hot gas in which the Sun is plunged. All these structures are believed to have been possibly triggered by supernova explosions taking place in UCL and LCC during the last 13 Myr (Maíz-Apellániz 2001).

The technique adopted for investigating the origins of the $\beta$ Pictoris Moving Group, for example, consists of tracing back the 3-D stellar orbits of the members of these moving groups

^ Table 1 is also available in electronic form at CDS via anonymous ftp to cdsarc.u-strasbg.fr $(130.79 .128 .5)$ or via http://cdsweb.u-strasbg.fr/cgi-bin/qcat?]/A+A/448/1001 until their main first orbits confinement was found, as well as the past mean positions of LCC and UCL. This enabled not only to determine the dynamical age of this moving group, but also to investigate properties of their birth clouds (Ortega et al. 2002, 2004). It is also possible to find the past positions of the possible supernovae that triggered the formation of these groups by tracing back the orbit of a runaway OB star, which could have been the result of a supernova explosion in LCC or UCL (see, for example, Hoogerwerf et al. 2001; and Vlemmings et al. 2004).

While the past evolution of these moving groups of low mass stars appears to be a relatively simple problem (as the dynamical ages are not so old), the dynamical evolution of the older and more numerous subgroups LCC and UCL appears to be more difficult. There is the possibility of the presence of several generations of hot stars during the mainstream of the OB association evolution (Garmany 1994). Substructure in LCC and UCL was found by de Bruijne (1999), based on Hipparcos data. The formation of the younger US subgroup could have been triggered by UCL some 6-8 Myr ago (Preibisch et al. 2001). All these studies require reliable radial velocities in order to calculate space velocities. In this paper we present single-epoch radial velocity $(R V)$ measurements for 56 B-type stars members of LCC, UCL and US subgroups, to contribute to studies of their dynamics so as to unravel their origins. 


\section{Observations and reduction}

A sample of 56 B-type stars from the Scorpius-Centaurus association was observed during observing runs in May 16-20 and July 7, 2002, with the $1.52 \mathrm{~m}$ telescope equipped with the FEROS echelle spectrograph (Kaufer et al. 2000; resolving power $R=48000$, wavelength coverage between 3900 and $9200 \AA$ ) with a CCD detector at the European Southern Observatory $(\mathrm{ESO})^{1}$. The target stars were selected from the list in Humphreys \& McElroy (1984) and from the comprehensive study of $\mathrm{OB}$ associations based on Hipparcos observations by de Zeeuw et al. (1999). The observed targets are listed in Table 1. From this sample, according to de Zeeuw et al. (1999), 15 targets are confirmed members of the LCC, while 15 stars are members of the UCL and 11 stars are from the US subgroup. For the remaining 15 stars in our sample, membership to any of these subgroups was not certain.

The spectra were reduced with the MIDAS reduction package and consisted of the following standard steps: CCD bias correction, flat-fielding, extraction, wavelength calibration, correction of barycentric velocity, as well as spectrum rectification and normalization. The one-dimensional spectra were then treated by tasks in the NOAO/IRAF data package. The signal-to noise ratio obtained in the observed spectra was typically larger than 100 and typical exposure times varied between $300 \mathrm{~s}$ for the brightest stars $(V \sim 3)$ and $1200 \mathrm{~s}$ for stars with $V \sim 5$. Typical spectra are shown in Fig. 1. The top panel corresponds to the target star HD 122980 and the bottom panel to HD 112092. Both stars have sharp lines with projected rotational velocities ( $v \sin i$ ) less than $40 \mathrm{~km} \mathrm{~s}^{-1}$. The spectral region displayed shows identifications of several lines that were used in the $R V$ determinations. The FEROS bench spectrograph and set up have proven to have high spectral stability for $R V$ measurements as concluded from a study of radial-velocity standard stars: a rms of $21 \mathrm{~m} \mathrm{~s}^{-1}$ has been obtained for a data set of 130 individual measurements (Kaufer et al. 2000).

\section{Analysis and discussion}

The cross-correlation technique, which is used for precise $R V$ determinations in later type stars, when applied to the hotter OB stars can be problematic as early type star spectra show few absorption lines. These lines are in many cases, intrinsically broad (up to a few hundreds $\mathrm{km} \mathrm{s}^{-1}$ ) due to stellar rotation. In addition, there is also the possibility of line variability affecting their line profiles (Steenbrugge et al. 2003). Therefore, the cross-correlation peak that defines the value of radial velocity can be very broad and contain important substructures caused by blending of spectral lines that appear to have different widths. In addition to having high $v \sin i$ values many OB stars are binary and it is not straightforward to apply the cross-correlation method and to identify them as doublelined binaries; in order to obtain the orbital solution a long set of observations is needed. Detailed cross-correlation technique analyses applied to determinations of radial velocities of early-type stars has been presented in a number of recent publications (see, for example, Verschueren et al. 1997, 1999a;

\footnotetext{
1 Observations obtained under the ON/ESO agreement.
}

Griffin et al. 2000). Griffin et al. (2000), in particular, discuss in detail the difficulties in obtaining accurate $R V$ measurements from cross-correlation in early-type stars spectra.

In this study, having high-resolution observations covering a large spectral range, radial velocity values for the target stars were obtained from measurements of the positions of individual spectral lines of He I, C II, N II, O II, Mg II, Si II and $\mathrm{Si}$ III, relative to their rest wavelengths. Radial-velocity standard stars were not observed. (The adopted linelists can be found in Daflon et al. 2001, 2003.) We inspected and identified all unblended lines visible in the spectral range between $3798 \AA(\mathrm{HI})$ and $7065 \AA$ ( $\mathrm{He} \mathrm{I})$ in each target star: the number of measurable lines varied between 10 and 74, depending on the star spectral type, rotation velocity, possible multiplicity, but also on the signal-to-noise of the obtained spectra. (We note, however, that for the double lined binary HD 133242, it was possible to measure positions only for 4 lines in component $\mathrm{A}$ and 6 lines in component B.) Mean radial velocities using all measurable lines $(R V)$ and respective dispersions were calculated for the individual target stars.

In Table 1 we assemble our $R V$ results as well as results from the literature. In the two first columns of this table we list the HD numbers of the observed stars with the respective spectral types; in the Cols. 3 and 4 we list the heliocentric Julian Date (HJD) and the measured radial velocities, plus the number of measured lines in brackets. In the other columns we list results from the literature: Cols. 5 and 6 list the $R V_{\mathrm{GCRV}}$ and associated error or quality, from the General Catalogue of Radial Velocities (GCRV; quality flags A to E, or I for insufficient data); Col. 7 presents the projected rotational velocity from Brown \& Verschueren (1997) and, when not available in this source, the $v$ sin $i$ was taken from the compilation of Glebocki \& Stawikowski (2000); in Col. 9 we list, when available, literature references where information about duplicity can be found for the stars. The stars are separated according to the different subgroups in the Scorpius-Centaurus association, following the membership probabilities $P(m)$ listed by de Zeeuw et al. (1999).

The internal precision of our $R V$ determinations can be represented by the scatter obtained from the $R V$ measurements line-by-line, which is listed in Col. 4 of Table 1. These are typically smaller than $\sim 2.0 \mathrm{~km} \mathrm{~s}^{-1}$ for stars with estimated $v$ sin $i$ smaller than $100 \mathrm{~km} \mathrm{~s}^{-1}$. We note, however, that when the target $v \sin i$ are large, the uncertainties in the derived $R V \mathrm{~s}$ can be significantly larger due to uncertainties in defining the line center. This can be seen in Fig. 2, where we show the obtained line-to-line scatter versus the target projected rotational velocity (as taken from Brown \& Verschueren 1997; and Glebocki \& Stawikowski 2000).

In order to evaluate possible systematic effects that line selection could have on the $R V$ results, we selected a homogeneous set of 28 spectral lines of H I, He I, Si III and Mg II, that could be measured in most of the studied spectra, and recalculated the mean radial velocity values for all possible stars. A comparison of the mean radial velocities $R V$ with $R V_{28}$ lines (obtained using only the selected 28 lines) indicates that there are non-significant systematic differences between 
Table 1. Radial velocities of observed Sco-Cen OB stars.

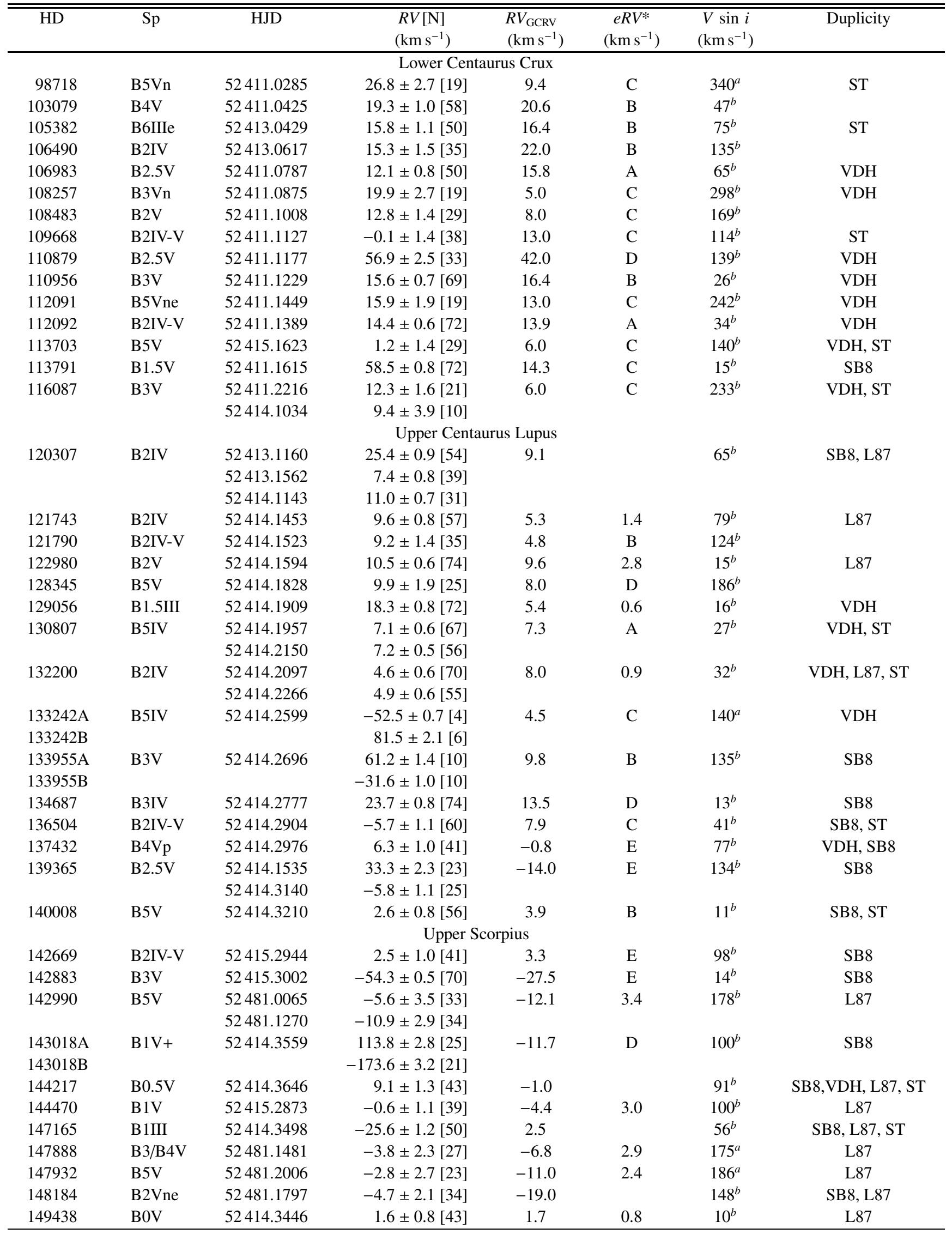


Table 1. continued.

\begin{tabular}{|c|c|c|c|c|c|c|c|}
\hline $\mathrm{HD}$ & $\mathrm{Sp}$ & HJD & $\begin{array}{c}R V[\mathrm{~N}] \\
\left(\mathrm{km} \mathrm{s}^{-1}\right)\end{array}$ & $\begin{array}{l}R V_{\mathrm{GCRV}} \\
\left(\mathrm{km} \mathrm{s}^{-1}\right)\end{array}$ & $\begin{array}{c}e R V^{*} \\
\left(\mathrm{~km} \mathrm{~s}^{-1}\right)\end{array}$ & $\begin{array}{c}V \sin i \\
\left(\mathrm{~km} \mathrm{~s}^{-1}\right)\end{array}$ & Duplicity \\
\hline \multicolumn{8}{|c|}{ Other } \\
\hline 104841 & B2IV & 52411.0594 & $-8.9 \pm 0.5[73]$ & 16.1 & I & $25^{b}$ & SB8 \\
\hline 105435 & B2IVne & 52411.0725 & $3.8 \pm 2.8[15]$ & 11.0 & $\mathrm{C}$ & $298^{b}$ & $\mathrm{VDH}$ \\
\hline 105937 & B3V & 52413.0558 & $22.7 \pm 1.5[33]$ & 15.0 & $\mathrm{C}$ & $129^{b}$ & \\
\hline 109026 & B5V & 52411.1069 & $4.0 \pm 1.6[31]$ & 2.5 & $\mathrm{D}$ & $188^{b}$ & \\
\hline 110335 & B6IVe & 52481.0996 & $4.8 \pm 1.8[23]$ & 12.5 & B & $250^{a}$ & \\
\hline 111123 & B0.5IV & 52411.1357 & $9.8 \pm 0.7[53]$ & 10.3 & A & $40^{b}$ & $\mathrm{VDH}$ \\
\hline 115846 & B3IV & 52481.97 & $-21.8 \pm 1.6[27]$ & 3.0 & 4.0 & $168^{b}$ & \\
\hline 116072 & $\mathrm{~B} 2.5 \mathrm{Vn}$ & 52411.1714 & $18.8 \pm 2.5[21]$ & 3.0 & $\mathrm{C}$ & $233^{b}$ & $\mathrm{VDH}$ \\
\hline 118716 & B1III & 52413.1107 & $14.0 \pm 1.2[32]$ & 3.0 & B & $114^{b}$ & VDH \\
\hline \multirow[t]{2}{*}{120640} & $\mathrm{~B} 2 \mathrm{Vp}$ & 52413.1209 & $-2.1 \pm 0.8[63]$ & -4.7 & 0.8 & $21^{b}$ & \\
\hline & & 52414.1219 & $-1.5 \pm 0.6[49]$ & & & & \\
\hline 126341 & B2IV & 52414.1712 & $-21.1 \pm 1.0[73]$ & -21.5 & B & $15^{b}$ & VDH \\
\hline 132058 & B2III & 52414.2050 & $0.1 \pm 1.0[48]$ & 0.2 & 0.9 & $92^{b}$ & L87 \\
\hline 132955 & B3V & 52414.2424 & $5.1 \pm 0.5[70]$ & 3.7 & 2.1 & $8^{b}$ & $\mathrm{VDH}$ \\
\hline 144218 & $\mathrm{~B} 2 \mathrm{~V}$ & 52414.3716 & $0.6 \pm 0.8[57]$ & -5.6 & 0.8 & $56^{b}$ & VDH, L87, ST, SB8 \\
\hline 151985 & B2IV & 52414.3352 & $1.9 \pm 0.7[56]$ & 1.3 & 0.7 & $52^{b}$ & L87, SB8 \\
\hline
\end{tabular}

* A: errors $\leq 2.5 \mathrm{~km} \mathrm{~s}^{-1}$; B: $2.5<$ errors $\leq 5.0 \mathrm{~km} \mathrm{~s}^{-1}$; C: $5.0<$ errors $\leq 10.0 \mathrm{~km} \mathrm{~s}^{-1}$; D: errors $\geq 10 \mathrm{~km} \mathrm{~s}^{-1}$; E: too uncertain (from Table 3 of Barbier-Brossat \& Figon 2000).

${ }^{a}$ From Glebocki \& Stawikowski (2000) and ${ }^{b}$ from Brown \& Verschueren (1997).

SB8 - Eighth Orbital Elements of Spectroscopic Binaries (Batten et al. 1989); L87 - Levato et al. (1987); VDH - Visual Double Stars in Hipparcos (Dommanget \& Nys 2000); ST - Shatsky \& Tokovinin (2002).

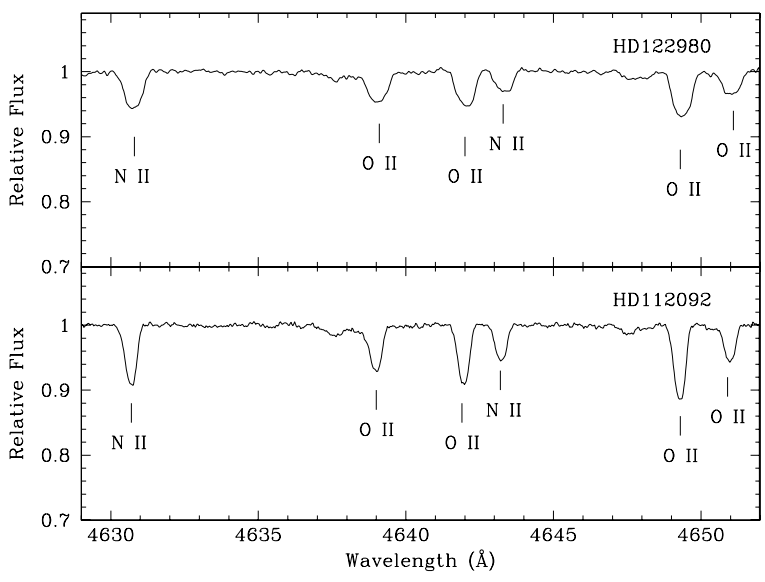

Fig. 1. Sample spectra for two target stars. The top panel shows HD 122980 and the bottom panel shows HD 112092. The lines appearing in this spectral region are identified.

the two determinations $R V-R V_{28 \text { lines }}=-1.0 \mathrm{~km} \mathrm{~s}^{-1}$, with $\sigma=3.1 \mathrm{~km} \mathrm{~s}^{-1}$.

\subsection{Membership}

Table 1 lists the target stars according to their membership the 3 subgroups as assigned by de Zeeuw et al. (1999). Most of the stars in the Lower Centaurus Crux subgroup are flagged as binaries in the literature, except for HD 103079, HD 106490

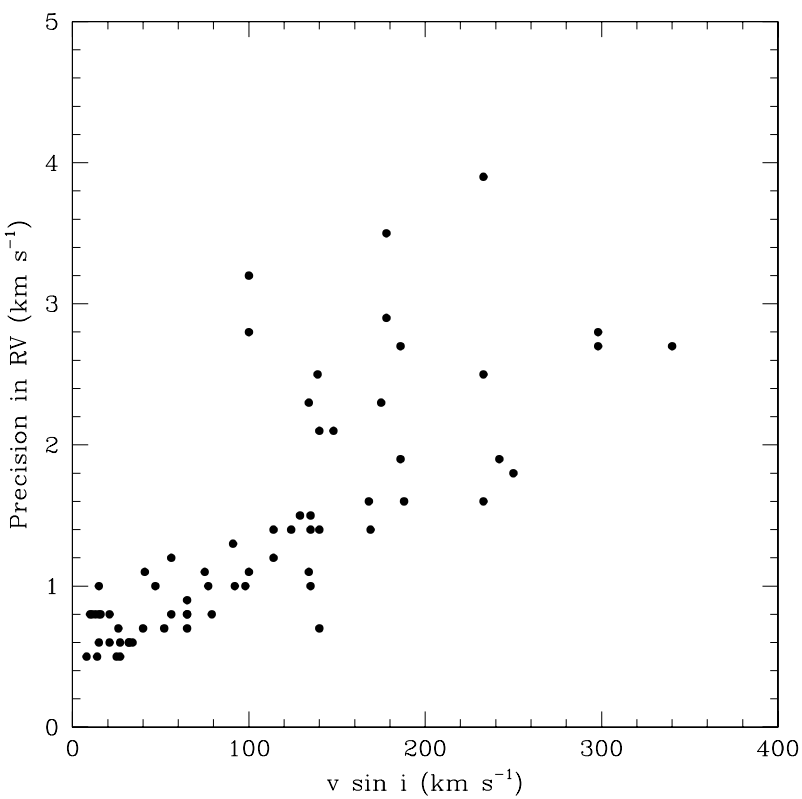

Fig. 2. The dependance of the internal rms errors of our radial velocity determinations on the star's projected rotational velocities $(v \sin i)$ taken from Brown \& Verschueren (1997) and Glebocki \& Stawikowski (2000).

and HD 108483. For these 3 stars we measured radial velocities of $19.3 \mathrm{~km} \mathrm{~s}^{-1}, 15.3 \mathrm{~km} \mathrm{~s}^{-1}$ and $12.8 \mathrm{~km} \mathrm{~s}^{-1}$, respectively, with $R V_{\text {mean }}=15.8 \pm 3.2 \mathrm{~km} \mathrm{~s}^{-1}$. This mean value is 
in general agreement with the mean radial velocity calculated by de Zeeuw et al. (1999) for LCC, which is of $12 \mathrm{~km} \mathrm{~s}^{-1}$. For the subgroup UCL, our sample has 2 non-binary stars (HD 121790 and HD 128345) and $R V_{\text {mean }}=9.6 \mathrm{~km} \mathrm{~s}^{-1}$ which is $\sim 5 \mathrm{~km} \mathrm{~s}^{-1}$ higher than the de Zeeuw et al. (1999) mean value of $4.9 \mathrm{~km} \mathrm{~s}^{-1}$. For the Upper Scorpius subgroup all the sample stars have been flagged as binaries in the literature.

For the five target stars that had not been identified as members of any of the three subgroups in the Sco-Cen association (listed as "others" in Table 1) and for which we have no information on duplicity, we can attempt to discuss their membership status based on the comparison of the radial velocities measured here and in the literature. We find that the measured radial velocities for HD $109026\left(R V=4.0 \mathrm{~km} \mathrm{~s}^{-1}\right)$ and HD $110335\left(R V=4.8 \mathrm{~km} \mathrm{~s}^{-1}\right)$ are consistent with the mean radial velocity for UCL of $4.9 \mathrm{~km} \mathrm{~s}^{-1} \mathrm{~s}$. For the target star HD 109026 we have $R V_{\mathrm{GCRV}}=2.5 \mathrm{~km} \mathrm{~s}^{-1}$, therefore it could be considered initially as having constant $R V$ (within the uncertainties) and possibly a member of the Upper Centaurus Lupus subgroup. For HD 110335, we find a larger discrepancy between our measurement $\left(R V=4.8 \mathrm{~km} \mathrm{~s}^{-1}\right)$ and the $R V$ value in the GCRV $\left(R V_{\mathrm{GCRV}}=12.5 \mathrm{~km} \mathrm{~s}^{-1}\right)$. The $R V \mathrm{~s}$, however, are marginally consistent given the expected uncertainty brackets that affect the 2 determinations. If this is really the case, HD 110335 could be also considered as a possible member of the UCL subgroup. In addition, the target star HD $120640\left(R V_{\text {mean }}=-1.8 \mathrm{~km} \mathrm{~s}^{-1}\right.$ from this study and $R V_{\mathrm{GCRV}}=-4.7 \mathrm{~km} \mathrm{~s}^{-1}$ ) can be assumed here to have constant radial velocity. These measurements are consistent with the mean $R V$ value of $-4.6 \mathrm{~km} \mathrm{~s}^{-1}$ listed by de Zeeuw et al. (1999) for this subgroup.

The two other stars in our sample of "others" (HD 115846 and HD 105937) for which we derived $R V=-21.8 \mathrm{~km} \mathrm{~s}^{-1}$ and $R V=22.7 \mathrm{~km} \mathrm{~s}^{-1}$, respectively, have values in the GCRV of $R V_{\mathrm{GCRV}}=3.0 \mathrm{~km} \mathrm{~s}^{-1}$ and $R V_{\mathrm{GCRV}}=15.0 \mathrm{~km} \mathrm{~s}^{-1}$. We found no information in the literature about these stars being confirmed binary stars, but the variation in $R V$ for HD 115846 exceeds the expected uncertainties: this target probably has a non-constant $R V$, which prevents further considerations about it belonging to any of the Sco-Cen subgroups. HD 105937 has an $R V$ only marginally constant within the uncertainties, but its mean $R V$ is not compatible with any of the subgroups.

\subsection{Duplicity}

Results from a search for duplicity information for the targets stars (Col. 9; Table 1) indicate that a large number of stars in our sample are flagged as binaries in the literature. For most of these targets we have only one single-epoch $R V$ measurement and our results alone cannot be used to infer duplicity. However, the $R V \mathrm{~s}$ derived in this study can be added to $R V$ databases and contribute to long term studies of their orbits. Only a small number of stars had not been previously flagged as binaries in the different studies in the literature. For this subsample of 10 stars, considered a priori as $R V$ constants, it is possible to compare our $R V$ values with the averaged radial velocities assembled in the GCRV. This comparison is shown in

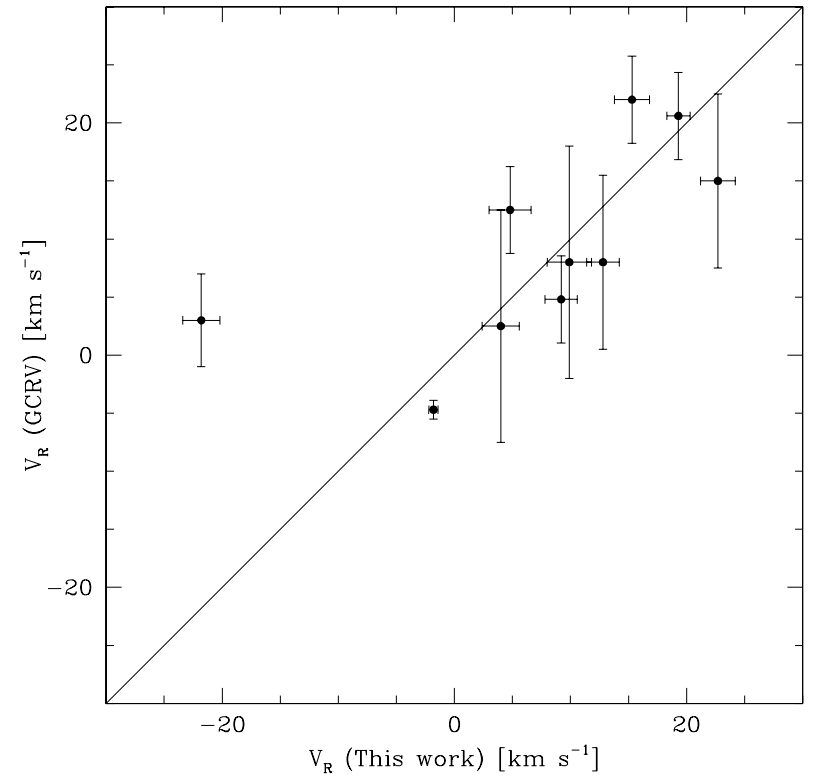

Fig. 3. A comparison between the radial velocities derived in this study with previously determined radial velocities from the GCRV (Barbier-Brossat \& Figon 2000). The targets shown are those stars from our sample considered to be single stars. The adopted error bars for this study are the sigmas listed in Col. 4 of Table 1. The adopted error bars from the GCRV were calculated as the mean value of the range of uncertainties in $R V$ corresponding to the quality flags A-D (listed in Table 1). For those stars with $R V$ quality $\mathrm{A}$ and $\mathrm{D}$ we adopted uncertainties of $2.5 \mathrm{~km} \mathrm{~s}^{-1}$ and $10 \mathrm{~km} \mathrm{~s}^{-1}$, respectively. The $x=y$ line representing perfect agreement is shown for comparison. The very discrepant point at $(-21.8,3.0)$ represents the target star HD 115846, which could be an unsuspected binary.

Fig. 3. Our $R V$ determinations compare favorably with the $R V \mathrm{~s}$ from the GCRV with a scatter of the order of the estimated uncertainties. $R V_{\mathrm{GCRV}}-R V=0.7$ and $\sigma=4.9 \mathrm{~km} \mathrm{~s}^{-1}$. (This was calculated excluding one discrepant star, HD 115846, which could be a binary system.) Taking into account the mean precision of $R V$ determinations from the GCRV as $\pm 5 \mathrm{~km} \mathrm{~s}^{-1}$, the external precision of our $R V$ determinations may be evaluated as approximately $\pm 5 \mathrm{~km} \mathrm{~s}^{-1}$.

For those targets with more than one epoch $R V$ measurement in our study, a subsample showed radial velocity variations larger than the expected uncertainties: HD 120307, HD 142990 and HD 139365. Since these had been previously identified as SBs in the literature (Levato et al. 1987; and Batten et al. 1989), our results confirm their duplicity. Four other stars with multiple epoch observations in this study showed a constant $R V$ within the uncertainties: HD 116087, HD 130807, HD 132200 and HD 120640.

For 3 targets in our sample (HD 133242, HD 133955 and HD 143018) we were able to separate and identify lines of two stellar components, classifying them as double lined spectroscopic binaries. Their combined spectra showing spectral lines from two stars are shown in Fig. 4. Two of these stars (HD 143018 and HD 133955) were previously identified in Batten et al. (1999) as a spectroscopic binaries. 


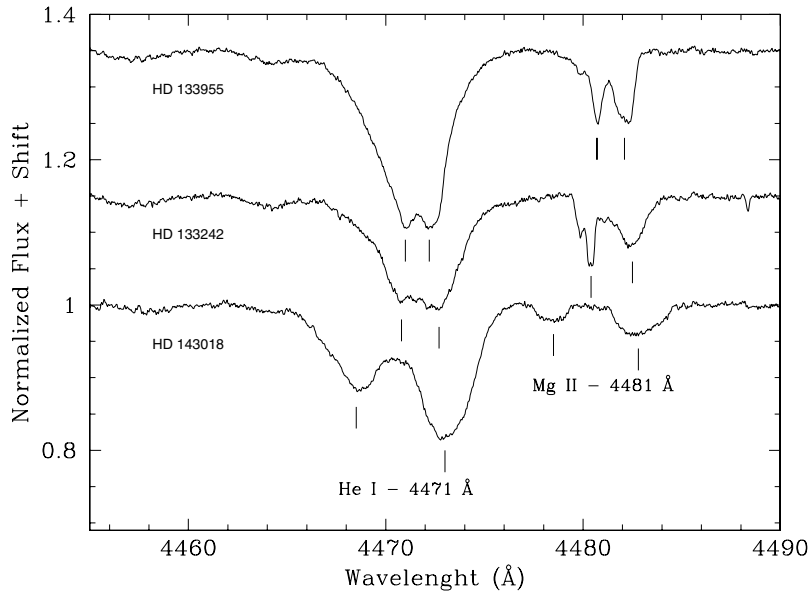

Fig. 4. Sample spectra of the double lined spectroscopic binaries HD 133242, HD 133955 and HD 143018 showing the lines $4471 \AA$ of He I and $4481 \AA$ of $\mathrm{Mg}$ II.

Acknowledgements. We thank the anonymous referee for suggestions that significantly improved the paper. E.G.J. thanks FAPERJ and MCT Brazil for financial support.

\section{References}

Barbier-Brossat, M., \& Figon, P. 2000, A\&AS, 142, 217

Batten, A. H., Fletcher, J. M., \& MacCarthy, D. G. 1989, Publications of the Dominion Astrophysical Observatory, 17, 1

Blaauw, A. 1960, Present Problems Concerning the Structure and Evolution of the Galactic System, ed. J. H. Oort, \& H. G. Quik, Nuffic Intern. Summer Course, 3

Blaauw, A. 1964, ARA\&A, 2, 213

Brown, A. G. A., \& Verschueren, W. 1997, A\&A, 319, 811

de Bruijne, Jos H. J. 1999, MNRAS, 310, 585 de Zeeuw, P. T., Hoogerwerf, R., de Bruijne, J. H. J., Brown, A. G. A., \& Blaauw, A. 1999, AJ, 117, 354

Daflon, S., Cunha, K., Becker, S., \& Smith, V. V. 2001, ApJ, 563, 325

Daflon, S., Cunha, K., Smith, V. V., \& Butler, K. 2003, A\&A, 399, 525

Dommanget, J., \& Nys, O. 2000, A\&A, 363, 991

Garmany, C. 1994, PASP, 106, 25

Glebocki, R., \& Stawikowski, A. 2000, A\&A, 50, 509

Griffin, R. E. M., David, M., \& Verschueren, W. 2000, A\&AS, 1407, 299

Hoogerwerf, R., de Bruijne, J. H. J., \& de Zeeuw, P. T. 2001, A\&A, 365,49

Humphreys, R. M., \& McElroy, D. B. 1984, ApJ, 284, 565

Jilinski, E., Ortega, V. G., \& de la Reza, R. 2005, ApJ, 619, 945

Kaufer, A., Stahl, O., Tubbesing, S., et al. 2000, Proc. SPIE V., 4008, 459

Levato, H., Malaroda, S., Morrell, N., \& Solivella, G. 1987, ApJS, 64, 487

Maíz-Apellániz 2001, ApJ, 560, L83

Mamajek, E. E., Meyer, M. R., \& Liebert, J. 2002, AJ, 124, 1670

Mamajek, E. E., Lawson, W. A., \& Feigelson, E. D. 2000, ApJ, 544, 356

Ortega, V. G., de la Reza, R., Jilinski, E., \& Bazzanella, B. 2002, ApJ, 575, L75

Ortega, V. G., de la Reza, R., Jilinski, E., \& Bazzanella, B. 2004, ApJ, 609, 243

Preibisch, T., Guenther, E., \& Zinnecker, H. 2001, AJ, 121, 1040

Sartori, M. J., Lepine, J. R. D., \& Dias, W. S. 2003, A\&A, 404, 913

Steenbrugge, K. C., de Bruijne, J. H. J., Hoogerwerf, R., \& de Zeeuw, P. T. 2003, A\&A, 402, 587

Shatsky, N., \& Tokovinin, A. 2002, A\&A, 382, 92

Verschueren, W., Brown, A. G. A., Hensberge, H., et al. 1997, PASP, 109,868

Verschueren, W., David, M., \& Griffin, R. E. M. 1999, A\&AS, 140, 107

Vlemmings, W. H. T., Cordes, J. M., \& Chatteriee, S. 2004, ApJ, 610, 402 